\title{
Assessment of quality of life in glaucoma patients using the Glaucoma Quality of Life-15 questionnaire
}

Pallak Kusumgar, Sulatha V. Bhandary, Lavanya Rao, Surabhi Abbagani, Krishna Rao Addoor

Department of Ophthalmology, Kasturba Medical College Manipal, Manipal Academy of Higher Education, Manipal, India

\begin{abstract}
Purpose: The purpose of this study was to assess quality of life in glaucoma patients using the Glaucoma Quality of Life-15 (GQL-15) questionnaire. The GQL-15 questionnaire is a glaucoma-specific quality of life questionnaire used to assess the degree of functional disability caused by glaucoma.

Design: Cross-sectional, prospective study with comparison group at a tertiary care hospital.

Material and methods: The GQL-15 questionnaire was used to evaluate the quality of 80 patients in the glaucoma group and 80 normal subjects in the control group. The data were analyzed by test of proportions and Chi-square test. A p-value of $<0.05$ was considered significant.

Results: Glaucoma patients (mean age: $60.5 \pm 9.2$ years; range: $43-86$ years) and comparison group (mean age: $58.9 \pm 4.5$ years; $52-72$ years) were matched with respect to age ( $p=0.18)$. Glaucoma patients had significantly worse quality of life compared to the non-glaucoma group $(p<0.001)$. Significant differences $(p<0.05)$ between the scores of the mild, moderate, and severe glaucoma groups were observed, suggesting poorer quality of life as the severity of glaucoma increases.

Conclusions: The GQL-15 revealed that quality of life worsens with increasing severity of glaucoma increases. This fact is important when educating patients about the disease course and its therapy.
\end{abstract}

Keywords: Glaucoma Quality of Life-15 (GQL-15) questionnaire, primary open-angle glaucoma, quality of life

\section{Introduction}

Glaucoma is a chronic, progressive, and irreversible disease resulting in severe visual disability, which has severe implications on the quality of life of affected

Correspondence: Dr. Sulatha V. Bhandary, Professor and Head, Department of Ophthalmology, Kasturba Medical College Manipal, Manipal Academy of Higher Education, Manipal, India.

E-mail: sulatha_bhandary@yahoo.co.in 
patients. Quality of life can be defined as subjective wellbeing, which depends on a patient's values, priorities, experiences, and aspirations, and reflects the difference between the hopes and expectations of a person and their experiences. Assessment of quality of life has been used to understand the impact of various factors linked to the disease process in a patient's life and this understanding has led to better outcomes of treatment. ${ }^{1}$ The various tools available for measuring quality of life can be generic or disease-specific. While there is no gold-standard quality of life assessment scale, glaucoma-specific and vision-specific instruments are better than generic tools to assess the impact of disease per se on the patients' overall wellbeing. There are a number of such well-documented tools, such as the Short Form-36 (SF-36) or Short Form-12 (SF-12), 25-item National Eye Institute Visual Function Questionnaire (NEI VFQ-25), Visual Activities Questionnaire (VAQ), Activities of Daily Vision Scale, and Glaucoma Quality of Life-15, (GQL-15) that have been used to quantify the subjective status of glaucoma patients. ${ }^{2}$ Our study aims to assess the quality of life in glaucoma patients by using the GQL-15 questionnaire.

\section{Methods}

The study was conducted in a tertiary care at the Kasturba Medical College Hospital (Manipal, Karnataka State, south India) from October 2012 to September 2014. The study was conducted after clearance from the Institutional Ethical Committee.

\section{Sample size}

A standard deviation of 8 and a clinically significant difference in the mean GQL-15 score of 5 among glaucoma patients and non-glaucoma comparison group was anticipated based on the pilot study. At $95 \%$ confidence level, for a power of $80 \%$, the calculated minimum sample size was 40 subjects with glaucoma and 40 non-glaucoma subjects. The present cross-sectional, prospective study was conducted at a tertiary care hospital, enrolling 80 patients who were diagnosed as primary open-angle glaucoma (POAG) and 80 non-glaucoma subjects who were healthy participants without any ocular pathology causing poor vision or family history of glaucoma.

Patients with an established diagnosis of POAG, on minimum of one antiglaucoma medications were included in the group of cases. Healthy age- and sex-matched individuals (above 40 years of age), with no family history of glaucoma, and who were able to speak and/or read English and/or the local regional language fluently were included in the group of controls. The subjects were briefed about the study and written informed consent was obtained from those willing to participate in the study. 
The exclusion criteria were: patients with other ocular diseases that could cause visual field defects or that led to poor performance on Humphrey Field Analyzer (HFA, (Carl Zeiss Meditec, Jena, Germany) test. Hence, patients with any non-glaucomatous condition or disease affecting visual function significantly, such as cataract, diabetic retinopathy, macular degeneration, retinal pathology, and non-glaucomatous optic neuropathy, were excluded. Patients suffering from any comorbidities severe enough to cause their own psychological morbidity and patients who were not fit to be subjected to clinical interview were also excluded from the study.

After enrollment, all the cases and controls were interviewed for demographic data comprising age, sex, occupation, and educational status. Patients were inquired about the number of topical antiglaucoma medications they were on, disease duration, and number of scheduled follow-up visits per year. All participants were subjected to general and systemic examination and asked for the detailed history of any systemic disease. A complete ophthalmic evaluation was done for all the participants, including visual acuity recorded using the Snellen chart, detailed anterior segment examination and IOP measurement according to standard protocol using calibrated Goldmann applanation tonometry (Haag Streit, Köniz, Switzerland). Gonioscopy was done to rule out any narrow angles. Detailed fundus examination under mydriasis was done by direct and indirect ophthalmoscopy examination and optic discs were evaluated using $90 \mathrm{D}$ with the slit lamp.

A visual field examination by the Humphrey Field Analyzer (C HFA 750 or 720), program 30-2 standard SITA (as per the department protocol) was performed on all the cases to assess the latest status of visual fields.

On the basis of severity of visual field loss, patients were divided into the following three categories using the Hodapp-Parrish-Anderson (HPA) criteria:

1. Early defect

- Mean deviation (MD) less than $-6 \mathrm{~dB}$

- Less than $25 \%$ of the points (18) are depressed below the $5 \%$ level and less than 10 points are depressed below the $1 \%$ level on the pattern deviation plot

- All points in the central $5^{\circ}$ must have a sensitivity of at least $15 \mathrm{~dB}$

2. Moderate defect

- $\quad$ MD less than $-12 \mathrm{~dB}$

- Less than $50 \%$ of the points (37) are depressed below the $5 \%$ level and less than 20 points are depressed below the $1 \%$ level on the pattern deviation plot

- No points in the central $5^{\circ}$ can have a sensitivity of $0 \mathrm{~dB}$

- Only one hemifield may have a point with sensitivity of $<15 \mathrm{~dB}$ within $5^{\circ}$ of fixation 
3. Severe defect (any of the following results)

- MD greater than $-12 \mathrm{~dB}$

- More than $50 \%$ of the points (37) are depressed below the $5 \%$ level or more than 20 points are depressed below the $1 \%$ level on the pattern deviation plot

- At least one point in the central $5^{\circ}$ has a sensitivity of $0 \mathrm{~dB}$

- Points within the central $5^{\circ}$ with sensitivity $<15 \mathrm{~dB}$ in both hemifields

After conducting the detailed eye evaluation, all the cases and controls were subjected to the GQL-15 questionnaire.

\section{GLQ-15 questionnaire}

The GLQ-15 is a glaucoma-specific questionnaire consisting of 15 items (Annex 1). Each question has scores ranging from 0 to 5 , where 0 is difficulty to perform the task due to non-visual problems, 1 is no difficulty, and 5 is severe difficulty. These 15 items are grouped into four subscales:

1. Factor 1: central and near vision (two items);

2. Factor 2: peripheral vision (six items);

3. Factor 3: dark adaptation and glare (six items); and

4. Factor 4: outdoor mobility (one item).

The highest score is 75 and the lowest is 15 . Higher scores represent more difficulty with vision-related activities and are associated with poorer quality of life.

\section{Results}

The age of the cases ranged from 43 to 86 years, with a mean age of $60.51 \pm 9.18$ years. The mean age among the three glaucoma severity groups was as follows: early: 57.9 years; moderate: 61.10 and severe 62.55 years. The age of the controls ranged from 42 to 72 years, with a mean age of $58.98 \pm 4.5$ years. The difference between the mean age of cases and controls was 1.53 , which was statistically not significant $(p=0.18)$.

The difference in the number of males and females in both the cases $(55 \%, n=$ 44 males) and controls (50\%, $n=40$ males) group was statistically not significant.

The educational level and working status of the cases and controls was noted. An educational level below Grade 10 was found in $40 \%(n=32)$ of cases and $46.2 \%$ $(n=37)$ of controls. The working status was divided into two groups: working and non-working, the latter of which included all those who were housewives/retired. The subjects in the non-working category were 37 (46.2\%) among the cases and $45(56.3 \%)$ among the controls.

The duration of diagnosis in each group of glaucoma severity was compared. The mean duration of diagnosis was highest in patients with severe glaucoma ( $p$ 
< 0.001). The details are as follows: $0-5$ years: 37 patients; $6-10$ years: 30 patients; 11-15 years: 10 patients; and > 15 years: 3 patients.

The number of eye drops used by the patients in the glaucoma groups is shown in Table 1. There were four patients in the severe group with four medications; two of them were not willing to undergo trabeculectomy and the other two were not fit for surgery.

The frequency of follow-up in the glaucoma groups were the following: 23 of 30 patients in the early group had a follow-up every year, 26 of 30 patients in the moderate group had a follow-up every 6 months, and 13 of 20 patients in the severe group had a follow-up every 3 months.

Table 1. Number of eye drops used by the patients in the glaucoma groups

\begin{tabular}{|l|l|l|l|}
\hline $\begin{array}{l}\text { Number of drops/ } \\
\text { day }\end{array}$ & $\begin{array}{l}\text { Early glaucoma } \\
(\boldsymbol{n}=\mathbf{3 0})\end{array}$ & $\begin{array}{l}\text { Moderate } \\
\text { glaucoma } \\
(\boldsymbol{n}=\mathbf{3 0})\end{array}$ & $\begin{array}{l}\text { Severe } \\
\text { glaucoma } \\
(\boldsymbol{n}=\mathbf{2 0})\end{array}$ \\
\hline 1 & 16 & 8 & 4 \\
\hline 2 & 14 & 18 & 2 \\
\hline 3 & 0 & 4 & 10 \\
\hline 4 & 0 & 0 & 4 \\
\hline
\end{tabular}

\section{GQL-15}

The mean GQL-15 score for glaucoma patients was 33.04, while for controls it was 14.36. The difference between the mean scores for cases and controls was found to be 18.67 at a $95 \%$ confidence interval of 16.6-20.7. This was statistically significant, with a $p$-value $<0.001$. The scores of each severity group of glaucoma patients were also compared. The mean GQL-15 score for early glaucoma patients was 23.13 , for moderate glaucoma patients was 34.9 , and for severe glaucoma patients was 45.1. The differences between the scores of all the glaucoma groups was statistically significant (Table 2). The difference between the GQL-15 score of controls compared to the early glaucoma group was also statistically significant $(p<0.001)$. Among glaucoma patients, duration of glaucoma diagnosis showed a strong correlation with higher GQL-15 scores, with a correlation coefficient of 0.6 (Fig. 1).

\section{Subscale GQL-15 analysis}

As detailed in the Methods section, the GQL-15 is divided into four main factors or subscales. The overall highest mean percentage score was for Factor 3: 49.7\%, followed by Factor 1: 46.38\%, Factor 2: 38.6\%, and Factor 4: 36.25\% (Table 3). 
Assessment of quality of life using the GQL-15

Table 2. Comparison of GQL-15 score amongst glaucoma severity groups

\begin{tabular}{|l|l|l|l|}
\hline & Mean difference & $\boldsymbol{p}$-value & Confidence interval \\
\hline Early-moderate & 11.76 & $<0.001$ & $10-13.5$ \\
\hline Early-severe & 21.9 & $<0.001$ & $19.9-23.9$ \\
\hline Moderate-severe & 10.2 & $<0.001$ & $8.2-12.1$ \\
\hline
\end{tabular}

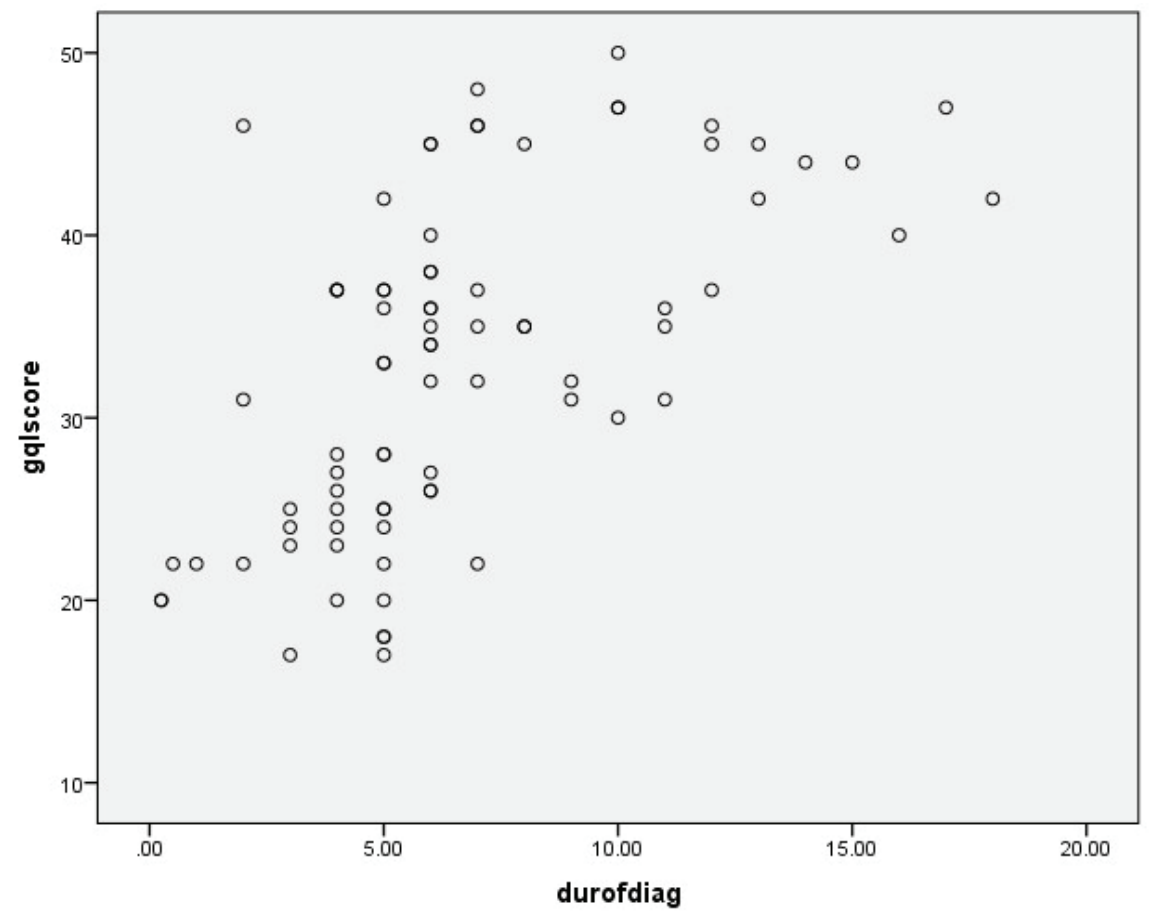

Fig. 1. Duration of diagnosis in relation to quality of life.

Correlations of the subscale analysis for each group of glaucoma severity showed that the highest score was for Factor 3 in early (37.4 \pm 5.9 standard deviation) and moderate $(53.6 \pm 4.9)$ glaucoma and for Factor 2 in severe glaucoma $(74.5 \pm 9.4)$.

\section{Effect of demographic factors on GQL-15}

The effect of factors such as age, gender, educational level, and working status was analyzed to evaluate whether they had on influence on the GQL-15 scores. 
Table 3. Overall GQL-15 scores of subscales in glaucoma cases

\begin{tabular}{|c|c|c|c|}
\hline Factors & $\begin{array}{l}\text { Minimum } \\
\text { \% score }\end{array}$ & $\begin{array}{l}\text { Maximum } \\
\text { \% score }\end{array}$ & Mean \% score ( \pm SD) \\
\hline $\begin{array}{l}\text { Factor } 1 \\
\text { Central and near vision }\end{array}$ & $20 \%$ & $90 \%$ & $46.38( \pm 21.5)$ \\
\hline $\begin{array}{l}\text { Factor } 2 \\
\text { Peripheral vision }\end{array}$ & $16 \%$ & $63 \%$ & $38.6( \pm 10.6)$ \\
\hline $\begin{array}{l}\text { Factor } 3 \\
\text { Glare and dark adaptation }\end{array}$ & $23 \%$ & $73 \%$ & $49.7( \pm 11.4)^{*}$ \\
\hline $\begin{array}{l}\text { Factor } 4 \\
\text { Outdoor mobility }\end{array}$ & $20 \%$ & $100 \%$ & $36.25( \pm 22.1)$ \\
\hline
\end{tabular}

*This was the factor with highest mean score.

Age

A positive correlation was seen between age and GQL-15 scores, with a correlation coefficient of 0.28 . This showed increasing GQL-15 scores with increasing age.

\section{Gender}

When gender was evaluated for its effect on GQL-15 score, it was observed that there was no significant statistical difference between males and females.

\section{Educational level and working status}

The patients who were educated above Grade 10 had similar GQL-15 scores to those with educational level below Grade 10. Both groups of working and non-working patients showed similar scores $(p>0.05)$ This indicated that educational level and working status had no effect on subjects' performance (Table 4).

\section{Discussion}

In glaucoma, disease progression and efficacy of treatment are evaluated by clinical indicators such as visual acuity, IOP level, perimetric findings, and treatment side effects. These are essentially objective measures to assess the disease. However, chronic diseases such as glaucoma lead to physical, psychological, and social dysfunction that affect the individual's quality of life.

Glaucoma affects quality of life in several ways. The diagnosis itself has a psychological impact, as the knowledge of suffering from a chronic and potentially blinding disease causes anxiety and fear in patients and their families. Glaucoma also causes functional disability as well as side effects and inconvenience due to treatment and follow-up. ${ }^{3,4}$

Demographic factors like age, gender, educational level, and working status 
Assessment of quality of life using the GQL-15

Table 4. Educational level and working status in relation to GQL-15 scores

\begin{tabular}{|l|l|l|l|l|}
\hline \multirow{2}{*}{} & \multicolumn{2}{|l|}{ Educational level } & \multicolumn{2}{l|}{ Working status } \\
\cline { 2 - 5 } & $>10^{\text {th }}$ Grade & $\begin{array}{l}<\mathbf{1 0}^{\text {th }} \\
\text { Grade }\end{array}$ & Working & Non-Working \\
\hline $\begin{array}{l}\text { Mean GQL-15 } \\
\text { scores }\end{array}$ & $24.13(11.2)$ & $23.13(11.6)$ & $23.4(10.3)$ & $23.9(12.2)$ \\
\hline Mean difference & 1.01 & 0.5 & \\
\hline $\boldsymbol{p}$-value & 0.5 & 0.7 & \\
\hline $\mathbf{9 5 \%} \mathrm{Cl}$ & $2.5-4.6$ & $3-4.1$ & \\
\hline
\end{tabular}

may also have an impact on quality of life. The Collaborative Initial Glaucoma Treatment Study (CIGTS) found age to be significant in determining quality of life. ${ }^{5}$ According to this study, younger subjects reported more problems than older age groups. This is probably due to the fact that expectations of health status decrease with increasing age. This study also showed gender to be an important factor, with females reporting more problems than males. In addition to this, patients with lower income reported more problems. ${ }^{6,7}$

Age was reported to have a negative impact on quality of life as measured by NEI VFQ-25 and GQL-15 in a study conducted on the Nigerian population, ${ }^{8}$ affecting all subscales of the GQL-15 and most subscales of the NEI VFQ-25. The same study also found women to have better vision-related quality of life scores ( $P=0.001$ for the NEI VFQ-25 and $P=0.005$ for the GQL-15).

In our study age, gender, educational level, and working status were studied to assess their impact on quality of life. Age was found to have a positive correlation with GQL-15 scores; thus, age had a negative impact on quality of life. In our study, males were found to have higher scores (poorer quality of life) compared to women, but this difference was not statistically significant.

In accordance with other studies, ${ }^{9-13}$ we also found a consistent pattern of worsening of functioning (higher GQL-15 scores) with increasing visual field loss correlating with glaucoma severity. In our study, the mean GQL-15 score for glaucoma patients was more than double compared to the age- and sex-matched controls, indicating a significant impact of disease on quality of life for glaucoma patients.

We divided glaucoma patients into three groups: early, moderate, and severe, based on the severity of visual field loss. Comparing the GQL-15 score of these groups, the scores obtained in the early glaucoma group were significantly different from the scores of moderate $(p<0.01)$ and advanced glaucoma ( $p<$ 0.001 ). A significant difference was also found between the moderate glaucoma group score and the advanced glaucoma group score $(p<0.01)$. 
In a study conducted on the Australian population by Goldberg et al. using the GQL -15 questionnaire, scores differed significantly among patients with mild, moderate, and severe glaucoma. ${ }^{9}$ Similar results were obtained by lester et al. using Viswanathan et al.'s questionnaire, where they found significant differences among the three groups. ${ }^{14}$ Vishal et al. used the 3-item Indian Vision Functioning Questionnaire (IND-VFQ-33) questionnaire, which revealed that newly diagnosed glaucoma patients have a significant worsening of quality of life after initiation of topical ocular hypotensive therapy. ${ }^{15}$

Four domains were analyzed for each glaucoma group in our study. The subscale scores afforded us the opportunity to examine loss of quality of life in the context of daily activities that may be especially troublesome for glaucoma patients. Factor 3, concerning glare and dark adaptation, was the most disabling domain for glaucoma patients in our study. This finding confirms the observations of Nelson et al. ${ }^{2}$ and the CIGTS, ${ }^{5}$ both of which also found these factors to be the most troublesome.' The Nigerian study also found glaucoma patients to have greatest difficulty with the glare and dark adaptation subscale of the GQL-15. ${ }^{8}$

The use of questionnaires to assess quality of life has advantages and disadvantages. A study by Kumar et al. assessed quality of life using verbally administered quality-of-life instruments comprising two glaucoma-specific instruments (GQL-15 and Viswanathan's 10-item instrument) and one vision-specific instrument (NEI VFQ-25) and found that all three questionnaires showed statistically significant correlation with decreased quality of life in glaucoma patients when compared to controls. ${ }^{19} \mathrm{~A}$ meta-analysis by Wang et al., where quality of life was measured using the GQL-15, concluded that quality of life for patients decreased as glaucoma severity increased. ${ }^{20}$

Despite the above-mentioned facts, the questionnaires do have pitfalls because they are subjective and therefore affected by various factors such as age, culture, language, gender, and education, among others. ${ }^{4,17,18,21}$ Performance-based measures, which involve testing what a person can and cannot do by actually observing the person attempting to perform specified tasks, are now gaining attention. $2,22,23$

The relatively large sample size, with the inclusion of participants with mild to severe visual field loss and their comparison with an equal number of age- and sex-matched controls, is one of the strengths of our study. Ours was a cross-sectional study carried out in tertiary healthcare center; hence, our sample may not be fully representative of the population and the results cannot be generalized to glaucoma patients in a community setting. We did not explore the potential effects of loss of color vision and other psychophysical measures. These psychophysical measures have been reported to be compromised in glaucoma. ${ }^{2}$ 
Several studies to assess quality of life in glaucoma patients were conducted on Western population. Comparatively, very few studies have been conducted on Indian populations using the GQL-15 questionnaire and ours is one of them.

\section{Conclusion}

The goal of glaucoma treatment should not be limited to controlling or reducing IOP to target level, but should also aim to provide patients with good functional vision to maintain an acceptable quality of life. Thus, with increasing awareness and rising importance to achieve a good QOL, patient-reported outcomes (subjective measures) are becoming increasingly important criteria to evaluate treatment efficacy. Proper counselling regarding the nature of the disease, its course, and a sound understanding of the problems faced by individual patients may be the key to successfully managing this chronic, debilitating, and potentially blinding disease.

The correlation observed between quality-of-life scores and objective measures of visual function suggests that inclusion of quality-of-life assessments in clinical practice could be highly informative to both patient and doctor. It would also help in providing patients with the best possible treatment, not only in terms of good vision, but also in maintaining or improving their overall quality of life. 


\section{Annex 1}

\section{GQL-15 QUESTIONNAIRE}

Does your vision give you any difficulty, even with glasses, with the following activities?

( 0 = abstinence from activity owing to non-visual reasons, $1=$ no difficulty, $2=$ a little bit of difficulty, $3=$ some difficulty, $4=$ quite a lot of difficulty, $5=$ severe difficulty)

1. Reading newspapers (Factor 1: Central and near vision)

2. Walking after dark (Factor 3: Dark adaptation and glare)

3. Seeing at night (Factor 3: Dark adaptation and glare)

4. Walking on uneven ground (Factor 2: Peripheral vision)

5. Adjusting to bright lights (Factor 3: Dark adaptation and glare)

6. Adjusting to dim lights (Factor 3: Dark adaptation and glare)

7. Going from light to dark room or vice versa (Factor 3: Dark adaptation and glare)

8. Tripping over objects (Factor 2: Peripheral vision)

9. Seeing objects coming from the side (Factor 2: Peripheral vision)

10. Crossing the road (Factor 4: Outdoor mobility)

11. Walking on steps/stairs (Factor 2: Peripheral vision)

12. Bumping into objects (Factor 2: Peripheral vision)

13. Judging distance of foot to step/curb (Factor 2: Peripheral vision)

14. Finding dropped objects (Factor 3: Dark adaptation and glare)

15. Recognizing faces (Factor 1: Central and near vision)

\section{References:}

1. Glen FC, Crabb DP, Garway-Heath DF. The direction of research into visual disability and quality of life in glaucoma. BMC Ophthalmol. 2011;11(1):19.

2. Nelson P, Aspinall P, Papasouliotis O, Worton B, O'Brien C. Quality of life in glaucoma and its relationship with visual function. J Glaucoma. 2003;12:139-150.

3. Heijl A, Traverso CE, editors. European Glaucoma Society Terminology and Guidelines for Glaucoma, 3rd ed. Italy; European Glaucoma Society: 2008. p. 119-120

4. lester M, Zingirian M. Quality of life in patients with early, moderate and advanced glaucoma. Eye. 2002;16(1):44-49.

5. Mills RP, Janz NK, Wren PA, et al. Correlation of visual field with quality-of-life measures of diagnosis in the Collaborative Initial Glaucoma Treatment study (CIGTS). J Glaucoma. 2001;10(3):192-198. 
6. Ramulu P. Glaucoma and disability: Which tasks are affected and at what stage of disease? Curr Opin Ophthalmol. 2009;20(2):92-98.

7. Janz NK, Wren PA, Lichter PR, Musch DC, Gillespie BW, Guire KE. Quality of life in newly diagnosed glaucoma patients: The Collaborative Initial Glaucoma Treatment Study. Ophthalmology 2001;108:887-97.

8. Onakoya AO, Mbadugha CA, Aribaba OT, Ibidapo OO. Quality of life of primary open angle glaucoma patients in Lagos, Nigeria: clinical and socio-demographic correlates. J Glaucoma. 2012;21(5):287-295.

9. Goldberg I, Clement Cl, Chiang TH, John WG, Lauren JL, Suart G, Paul H. Assessing quality of life in patients with glaucoma using the Glaucoma Quality of Life-15 (GQL-15) Questionnaire. J Glaucoma. 2009;18:6-12.

10. Skalicky S, Goldberg I. Depression and quality of life in patients with glaucoma: a cross-sectional analysis using the Geriatric Depression Scale-15, assessment of function related to vision, and the Glaucoma Quality of Life-15. J Glaucoma. 2008;17:546-551.

11. Van Gestel A, Webers $C A$, Beckers $H J$, et al. The relationship between visual field loss in glaucoma and health-related quality-of life. Eye. 2010;24:1759-1769.

12. Hirneiss C. Measurement of glaucoma-specific functionality with the GQL-15 and correlation with parameters of visual function. Ophthalmology. 2011;108(10):939-946.

13. Wang $B$, Aung $T$, Marella $M$, et al. Impact of bilateral open and closed-angle glaucoma on glaucoma-specific functioning in Asians. J Glaucoma. 2013;22(4):330-335.

14. lester M, Zingirian M. Quality of life in patients with early, moderate and advanced glaucoma. Eye. 2002;16:44-49.

15. Arora V, Bali SJ, Gupta SK, Vashisht P, Agarwal T, Sreenivas V, Dada TI. Impact of initial topical medical therapy on short-term quality of life in newly diagnosed patients with primary glaucoma. Indian J Ophthalmol. 2015;63:511-515.

16. Emerick GT. Quality of life and glaucoma medications. Do our prescriptions make a difference? Glaucoma Today. 2005;5:38-39.

17. Robin AL, Covert D. Does adjunctive glaucoma therapy affect adherence to the initial primary therapy? Ophthalmology. 2005;112(5):863-868.

18. Nordmann JP, Auzanneau N, Ricard S, Berdeaux G. Vision related quality of life and topical glaucoma treatment side effects. Health and Quality of Life Outcomes. 2003;1:75.

19. Kumar S, Ichhpujani P, Singh R, Thakur S, Sharma M, Nagpal N. The impact of primary open-angle glaucoma: Quality of life in Indian patients. Indian J Ophthalmol. 2018;66(3):416-419.

20. Wang Y, Alnwisi S, Ke M. The impact of mild, moderate, and severe visual field loss in glaucoma on patients' quality of life measured via the Glaucoma Quality of Life-15 Questionnaire: A meta-analysis. Medicine (Baltimore). 2017;96(48):e8019.

21. Varma R, Richman EA, Ferris FL, Bressler NM. Use of patient reported outcomes in medical product development: a report from the 2009 NEI/FDA Clinical Trial Endpoints Symposium. Invest Ophthalmol Vis Sci. 2010;51:6095-6103.

22. Khadka J, Pesudovs K, McAlinden C, Vogel M, Kernt M, Hirneiss C. Reengineering the glaucoma quality of life-15 questionnaire with Rasch analysis. Invest Ophthalmol Vis Sci. 2011;52(9):6971-6977.

23. Nelson $\mathrm{P}$, Aspinall PA, O'Brien C. Patients' perception of visual impairment in glaucoma: a pilot study. Br J Ophthalmol. 1999;83:546-552. 